Article

\title{
Correlative Experimental and Theoretical Investigation of the Angle-Resolved Composition Evolution of Thin Films Sputtered from a Compound $\mathrm{Mo}_{2} \mathrm{BC}$ Target
}

\author{
Jan-Ole Achenbach ${ }^{1} * \mathbb{D}$, Stanislav Mráz ${ }^{1}$, Daniel Primetzhofer ${ }^{2}$ and Jochen M. Schneider ${ }^{1}$ \\ 1 Materials Chemistry, RWTH Aachen University, Kopernikusstr. 10, 52074 Aachen, Germany; \\ mraz@mch.rwth-aachen.de (S.M.); schneider@mch.rwth-aachen.de (J.M.S.) \\ 2 Department of Physics and Astronomy, Uppsala University, Lägerhyddsvägen 1, 75120 Uppsala, Sweden; \\ daniel.primetzhofer@physics.uu.se \\ * Correspondence: achenbach@mch.rwth-aachen.de; Tel.: +49-241-80-25997
}

Received: 1 March 2019; Accepted: 20 March 2019; Published: 22 March 2019

\begin{abstract}
The angle-resolved composition evolution of Mo-B-C thin films deposited from a $\mathrm{Mo}_{2} \mathrm{BC}$ compound target was investigated experimentally and theoretically. Depositions were carried out by direct current magnetron sputtering (DCMS) in a pressure range from 0.09 to $0.98 \mathrm{~Pa}$ in $\mathrm{Ar}$ and $\mathrm{Kr}$. The substrates were placed at specific angles $\alpha$ with respect to the target normal from 0 to $\pm 67.5^{\circ}$. A model based on TRIDYN and SIMTRA was used to calculate the influence of the sputtering gas on the angular distribution function of the sputtered species at the target, their transport through the gas phase, and film composition. Experimental pressure- and sputtering gas-dependent thin film chemical composition data are in good agreement with simulated angle-resolved film composition data. In Ar, the pressure-induced film composition variations at a particular $\alpha$ are within the error of the EDX measurements. On the contrary, an order of magnitude increase in $\mathrm{Kr}$ pressure results in an increase of the Mo concentration measured at $\alpha=0^{\circ}$ from 36 at. $\%$ to 43 at. $\%$. It is shown that the mass ratio between sputtering gas and sputtered species defines the scattering angle within the collision cascades in the target, as well as for the collisions in the gas phase, which in turn defines the angle- and pressure-dependent film compositions.
\end{abstract}

Keywords: physical vapor deposition; $\mathrm{Mo}_{2} \mathrm{BC}$; Monte Carlo simulation; scattering; density functional theory

\section{Introduction}

$\mathrm{Mo}_{2} \mathrm{BC}$ is classified as a nanolaminated material with an orthorhombic structure [1-3]. It shows a unique combination of mechanical properties, such as an elastic modulus of $470 \mathrm{GPa}$, a ratio of bulk and shear moduli of 1.73, and a positive Cauchy pressure, which are required for hard and wear-resistant coatings with moderate ductility [3,4]. Bolvardi et al. [5] successfully synthesized crystalline $\mathrm{Mo}_{2} \mathrm{BC}$ at $380^{\circ} \mathrm{C}$ by high power pulse magnetron sputtering (HPPMS) [6] compared to a required temperature of $580^{\circ} \mathrm{C}$ during direct current magnetron sputtering (DCMS) [7]. The lower deposition temperature for the synthesis of a crystalline thin film by HPPMS was attributed to a larger adatom mobility induced by ion bombardment during HPPMS.

There are several synthesis approaches for the deposition of compound thin films, such as the utilization of reactive gases [7], co-sputtering from several targets [8,9], or targets with plugs [10]. Likewise, the employment of multi-elemental powder metallurgical composite targets is of great interest from an industrial application point of view due to the enhanced stability and repeatability [11] 
of non-reactive sputtering compared to reactive sputtering processes. However, it has been shown that the chemical composition of thin films deposited from multi-element targets deviates from the target composition, especially in targets with significant mass differences between their constituents, such as TiB [12-14], TiW [15-22], WB [23], SiC [24], MoSi [25,26], VC [27], NbC [28], Cr-Al-C [29,30], $\mathrm{Ti}_{2} \mathrm{AlC}$ [31], $\mathrm{Ti}_{3} \mathrm{SiC}_{2}$ [32,33], and CuZnSnSe [34]. The difference in the chemical film composition was attributed to several mechanisms: (i) the mass and size differences of the target constituents and the respectively associated different angular and energy distribution functions (EDF) of the sputtered species $[12,25,26,29,35]$; (ii) their mean free paths, as well as the energy transfer in collisions with the sputtering gas during transport $[12,13,15,21,29,35]$; and (iii) different sticking coefficients and re-sputtering of the film constituents by backscattered Ar [15-20].

The compositional evolution of binary Ti-B thin films was investigated experimentally and with a Monte Carlo model based on TRIDYN (dynamic transport of ions in matter) and TRIM (transport of ions in matter) codes [12]. It was shown that the Ti/B ratio strongly depends on the gas pressure and target-substrate distance, which in a product is proportional to the number of collisions sputtered species experience within the gas phase. The model was extended to $\mathrm{Cr}-\mathrm{Al}-\mathrm{C}$ thin films-a ternary system [29].

Van Aeken et al. [36] developed a Monte Carlo code SIMTRA for the simulation of sputtered particle trajectories in a gas-phase within a definable 3D setup. Collision modelling by interatomic potentials and thermal motion of background atoms are included within the code.

From the above, it can be learned that the deviation of the chemical composition of a thin film and multi-element target can be controlled by the sputtering pressure and gas type.

Within this work, experimental data were compared to a model based on TRIDYN and SIMTRA utilized for Mo-B-C thin films to understand how the gas phase transport affects the thin film chemical composition in a system with large mass differences of the multi-element target constituents.

\section{Materials and Methods}

\subsection{Experimental Details}

Mo-B-C thin films were deposited in a high vacuum chamber assembled from a DN160 six-way cross. A base pressure of $<1.1 \times 10^{-4}$ Pa was achieved before all depositions with a combination of a rotary-vane (Edwards E2M28, Edwards, Burgess Hill, UK) and a turbomolecular pump (Pfeiffer Vacuum TPU 240, Aßlar, Germany). A self-built magnetron with $\varnothing 90 \mathrm{~mm}$ was placed in the center of the chamber. A $6 \mathrm{~mm}$ thick $\mathrm{Mo}_{2} \mathrm{BC}$ compound target (Plansee Composite Materials $\mathrm{GmbH}$, Lechbruck am See, Germany) with the composition of 54.3 at.\%, 24.2 at.\%, and 21.5 at.\% of Mo, B, and C, respectively, bonded on a $\mathrm{Cu}$ backing-plate, was utilized for the investigations. The target contained a major $\mathrm{Mo}_{2} \mathrm{BC}$ phase with minor $\mathrm{Mo}_{2} \mathrm{C}$ and $\mathrm{MoC}$ phases (Figure 1), as measured by a Bruker D8 Discovery general area detector diffraction system (GADDS, Bruker, Billerica, MA, USA) with $\mathrm{Cu}(\mathrm{K} \alpha)$ radiation at $40 \mathrm{kV}$ and $40 \mathrm{~mA}$ with a constant incident angle of $\omega=15^{\circ}$.

The thin films were deposited for $1 \mathrm{~h}$ onto grounded, not intentionally heated $\mathrm{Si}$ (100) substrates with a size of approximately $15 \times 15 \mathrm{~mm}^{2}$ arranged at different angular positions with respect to the target normal of $\alpha \in\left\{0^{\circ}, \pm 22.5^{\circ}, \pm 45^{\circ}\right.$ and $\left.\pm 67.5^{\circ}\right\}$ (Figure 2). The target-substrate distance was kept constant at $70 \mathrm{~mm}$ with respect to the target center point. The DC power of $100 \mathrm{~W}$ was applied by an ADL $1.5 \mathrm{~kW}$ DC power supply (ADL Analoge und Digitale Leistungselektronik GmbH, Darmstadt, Germany). The Ar and Kr pressures utilized in the depositions are summarized in Table 1. 


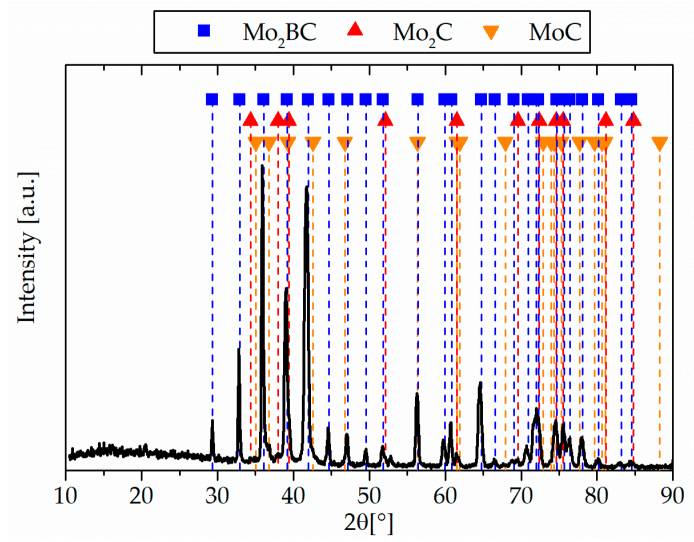

Figure 1. $\mathrm{XRD}$ pattern of the powder-metallurgically manufactured $\mathrm{Mo}_{2} \mathrm{BC}$ compound target. Small phase fractions of $\mathrm{Mo}_{2} \mathrm{C}$ and $\mathrm{MoC}$ were detected.

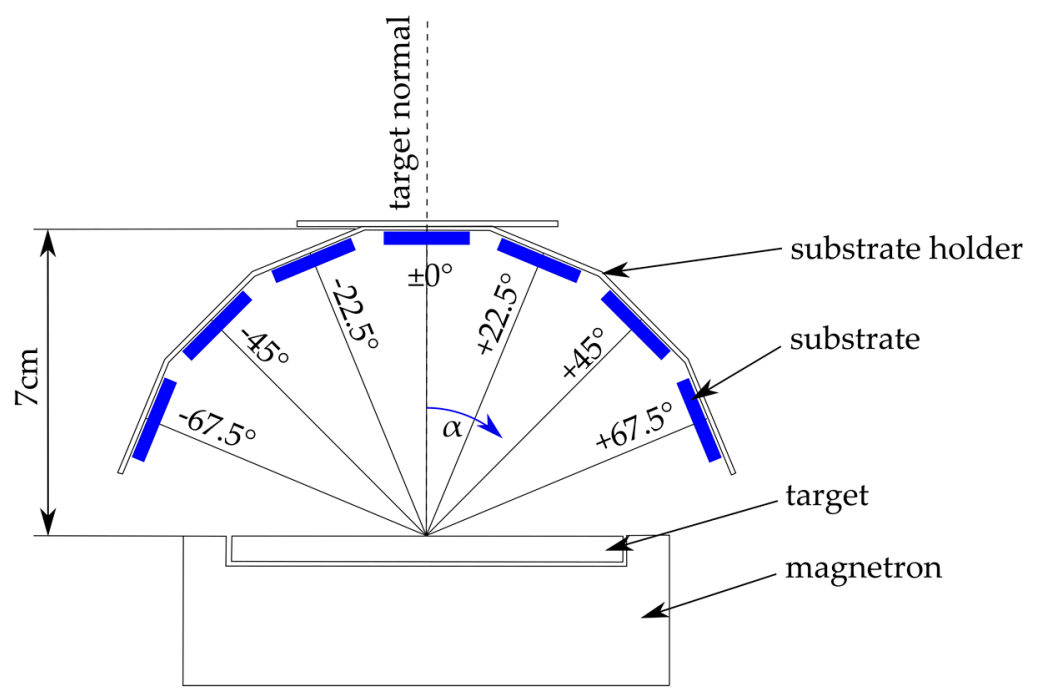

Figure 2. Experimental setup with seven substrates positioned at $\alpha \in\left\{0^{\circ}, \pm 22.5^{\circ}, \pm 45^{\circ}\right.$ and $\left.\pm 67.5^{\circ}\right\}$ angle arrangement with respect to the target normal and a target-substrate distance of approximately $70 \mathrm{~mm}$.

Table 1. Ar and $\mathrm{Kr}$ gas pressures and measured target voltages which correspond to impinging ion energies for $\mathrm{Ar}^{+}$and $\mathrm{Kr}^{+}$ions.

\begin{tabular}{cccc}
\hline \multicolumn{2}{c}{ Argon } & \multicolumn{2}{c}{ Krypton } \\
\hline Pressure (Pa) & Voltage (V) & Pressure (Pa) & Voltage (V) \\
\hline 0.09 & 401 & 0.09 & 441 \\
0.27 & 344 & 0.26 & 423 \\
0.46 & 328 & 0.45 & 421 \\
0.66 & 324 & 0.64 & 418 \\
0.98 & 314 & 0.96 & 403 \\
\hline
\end{tabular}

The chemical composition of the deposited films was measured by energy dispersive X-ray spectroscopy (EDX) attached to a JEOL JSM-6480 scanning electron microscope (SEM, JEOL, Tokyo, Japan). The electron gun of the SEM was operated at an acceleration voltage of $5 \mathrm{kV}$. Each sample was measured 10 times. The statistical uncertainty associated with this EDX quantification of Mo, B, and C was less than or equal to $5 \%$ relative deviation. To overcome the unknown systematic uncertainty for light elements in EDX, the samples deposited at $0.66 \mathrm{~Pa}$ Ar with $\alpha=0^{\circ},-22.5^{\circ},-45^{\circ}$, and $-67.5^{\circ}$ were quantified by time-of-flight elastic recoil detection analysis (ToF-ERDA) and used as a standard for the 
respective positions. The statistical uncertainty for all ToF-ERDA was $<0.4 \%$ absolute. In ToF-ERDA, the relative systematic uncertainties in the specific energy loss of the constituents and primary ions of the target are assumed to range from $5 \%$ to $10 \%$. Hence, the lower bound of the total measurement uncertainty for the EDX analysis with ToF-ERDA quantified standards ranges from $7 \%$ to $11 \%$.

\subsection{Simulation Details}

The angular-resolved chemical composition of the thin films was simulated with a Monte Carlo model based on TRIDYN [37,38] and SIMTRA [36] for the sputtering process and the gas phase transport, respectively.

\subsubsection{TRIDYN}

The impinging ion energies of $\mathrm{Ar}^{+}$and $\mathrm{Kr}^{+}$ions in the TRIDYN simulation were set according to the experimentally measured target voltages (Table 1 ). To address the dependence of the surface binding energy from the surface chemistry, a matrix model was introduced [38] and modified [29] for a system containing three elements, as presented in Equation (1), where $\mathrm{SBE}_{i}$ is the surface binding energy of the $i$-th target element at a given target concentration $c, c_{i}$ is the concentration of the $i$-th target element, and $\mathrm{SBV}_{i-j}$ is the surface binding potential of the $i$-th and $j$-th elements. $\mathrm{SBV}_{i-j}$ are assumed to be constant. Calculated angular distribution functions (ADF) and energy distribution functions (EDF) of the sputtered species are utilized in SIMTRA.

$$
\left(\begin{array}{c}
\mathrm{SBE}_{\mathrm{Mo}} \\
\mathrm{SBE}_{\mathrm{B}} \\
\mathrm{SBE}_{\mathrm{C}}
\end{array}\right)=\left(\begin{array}{ccc}
\mathrm{SBV}_{\mathrm{Mo}-\mathrm{Mo}} & \mathrm{SBV}_{\mathrm{Mo-}} & \mathrm{SBV}_{\mathrm{Mo}-\mathrm{C}} \\
\mathrm{SBV}_{\mathrm{B}-\mathrm{Mo}} & \mathrm{SBV}_{\mathrm{B}-\mathrm{B}} & \mathrm{SBV}_{\mathrm{B}-\mathrm{C}} \\
\mathrm{SBV}_{\mathrm{C}-\mathrm{Mo}} & \mathrm{SBV} V_{\mathrm{C}-\mathrm{B}} & \mathrm{SBV}_{\mathrm{C}-\mathrm{C}}
\end{array}\right)\left(\begin{array}{c}
\mathrm{C}_{\mathrm{Mo}} \\
\mathrm{c}_{\mathrm{B}} \\
\mathrm{c}_{\mathrm{C}}
\end{array}\right)
$$

For the determination of the surface binding potentials, an approach based on the energy conservation law $[29,38]$ was used and will in the following be called the energy conservation law approach. In addition, a DFT ab initio-based approach has been employed.

\subsubsection{Energy Conservation Law Approach}

The surface binding potential of pure elements $\mathrm{SBV}_{i-i}$ is assumed to be equal to the enthalpy of sublimation $\Delta_{\text {sub }} H_{i}$. The surface binding potential of the atom pairs $\mathrm{SBV}_{i-j}$ is calculated using Equation (2), where $\Delta_{\mathrm{f}} H_{\mathrm{Mo}_{\mathrm{n}} \mathrm{B}_{\mathrm{m}} \mathrm{C}_{\mathrm{o}}}$ is the enthalpy of formation of the ternary compound and $a$ and $b$ are the stoichiometric factors of the elements $i$ and $j$.

$$
\mathrm{SBV}_{i-j}=\frac{1}{2}\left(\Delta_{\mathrm{sub}} H_{i}+\Delta_{\mathrm{sub}} H_{j}\right)-\frac{1}{3} \frac{\mathrm{n}+\mathrm{m}+\mathrm{o}}{2 a b} \Delta_{\mathrm{f}} H_{\mathrm{Mon}_{\mathrm{n}} \mathrm{B}_{\mathrm{m}} \mathrm{C}_{\mathrm{o}}}
$$

The energy of formation per formula unit (f.u.) of $\Delta_{\mathrm{f}} H_{\mathrm{Mo}_{2} \mathrm{BC}}=-1.132 \frac{\mathrm{eV}}{\mathrm{f} . u .}$. used in the simulations was calculated by Bolvardi et al. [4]. The enthalpies of sublimation of $6.83,5.73$, and $7.51 \mathrm{eV}$ for Mo, B, and $C$ are given in the elements.dat file of TRIDYN, respectively. In addition, enthalpies of sublimation of $6.81,5.75$, and $7.37 \mathrm{eV}$ for Mo, B, and C, respectively, can be found in [39].

\subsubsection{Ab Initio Approach}

In addition to the TRIDYN approach, an ab initio approach based on DFT was used for the determination of the respective surface binding potentials. DFT calculations were implemented within the Vienna ab initio simulation package (VASP) [40,41]. Perdew-Burke-Ernzerhof (PBE) adjusted generalized gradient approximation (GGA) [42] was used for all calculations with projector augmented wave potential [43]. In addition, the tetrahedron method for total energy using Blöchl-corrections [44] and the reciprocal space integration using the Monkhorst-Pack scheme [45] were applied. The utilized $k$-point grid was $4 \times 4 \times 4$ for the (100) and (001) surfaces and $6 \times 2 \times 6$ for the (010) surface. The cut-off energy was set to $500 \mathrm{eV}$ with an electronic relaxation convergence of $0.01 \mathrm{meV}$. 
Considering the matrix model presented in Equation (1), the energy required to remove atoms of specific surfaces with different chemical compositions needs to be calculated. (100) and (001) surfaces, as well as different surface terminations of the (010) surface, are considered in the calculation and are illustrated in Figure 3. Subsequently, atoms are removed from the surface, creating a vacancy. The change in energy is considered to be the surface binding potential of the atom within the respective surface, as shown in Equation (3). $E_{i}$ is the energy of the atom $i$ after being removed from the surface, $E_{\mathrm{vac}, i}^{\text {surface } i}$ is the energy of surface $j$ with the vacancy of atom $i$, and $E^{\text {surface } j}$ is the energy of surface $j$ without a defect. Within DFT, the surfaces were simulated by a vacuum layer on top of the unit cell with the height of approximately $10 \AA$ for (100) and (001) and $17 \AA$ for (010) surfaces. Calculated SBVs for both approaches are presented in Equations (4) and (5).

$$
\begin{gathered}
\mathrm{SBV}_{i-j}=E_{i}+E_{\mathrm{vac}, i}^{\text {surface } j}-E^{\text {surface, } j} \\
\mathrm{SBV}_{\text {energy conservation law }}=\left(\begin{array}{lll}
6.83 & 6.66 & 7.50 \\
6.66 & 5.73 & 7.32 \\
7.50 & 7.32 & 7.41
\end{array}\right) \mathrm{eV} \\
\mathrm{SBV}_{\mathrm{ab} \text { initio }}=\left(\begin{array}{lll}
7.25 & 7.33 & 9.46 \\
7.19 & 6.98 & 9.71 \\
7.36 & 7.26 & 9.46
\end{array}\right) \mathrm{eV}
\end{gathered}
$$

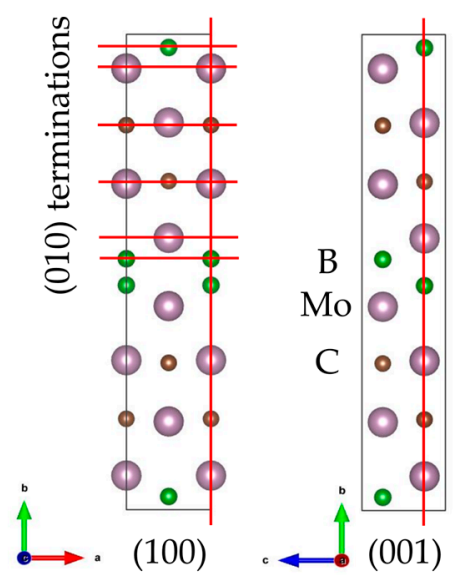

Figure 3. Considered (100), (001) surfaces and (010) surface terminations for the determination of the surface binding potentials in the ab initio approach. The colored spheres represent Mo atoms in purple, $\mathrm{B}$ atoms in green, and $\mathrm{C}$ atoms in brown. The figure was made with VESTA [46].

\subsubsection{SIMTRA}

Within SIMTRA simulations, $1 \times 10^{7}$ particles for Mo and $5 \times 10^{6}$ particles for B and C corresponding to a 2:1:1 target composition were transported. For the simulation setup, a cylinder with a diameter of $0.16 \mathrm{~m}$ and a length of $0.334 \mathrm{~m}$ was used. The target was positioned in the center of the simulation chamber. Seven circular substrates with a radius of $5 \mathrm{~mm}$ were arranged in the chamber corresponding to the actual experimental setup. The gas temperature was set to $300 \mathrm{~K}$. The atomic interaction was described with the Lenz-Jensen screening function implemented in SIMTRA. Gas motion and diffusion is considered within the gas transport. The racetrack profile of the target used for the experimental work was measured by a profilometer and taken into account for the simulations. The simulations were carried out in vacuum $\left(p_{\mathrm{Ar}}=1 \times 10^{-9} \mathrm{~Pa}\right)$ and in $\mathrm{Ar}$ and $\mathrm{Kr}$ gaseous atmosphere at pressures utilized in the experiments (Table 1). Atoms redeposited on the target during deposition are not sputtered again within the simulation. To overcome this virtual loss of particles, atoms redeposited on the target are distributed on all surfaces within the utilized 
simulation chamber with respect to the initial particle distribution, including the influence of the angular distribution function. For this, the ratio of deposited atoms on a substrate divided by the total number of sputtered atoms was multiplied by the number of deposited atoms on the target surface and added to the specific substrate.

\section{Results and Discussion}

\subsection{Experiment}

The angle- and pressure-dependent film compositions for both sputtering gases, $\mathrm{Ar}$ and $\mathrm{Kr}$, are presented in Figure 4. The target composition is indicated by black solid lines. For both sputter gases, the angle-dependence of Mo is convex, while the lighter elements B and C show a concave angle-dependence. At $\alpha \leq 22.5^{\circ}$ (Figure 2), a deficiency of the heavy element (Mo) and a surplus of light elements (B and C) is measured. Mo exhibits a deficiency of up to 18 at.\%, while B and $\mathrm{C}$ exhibit a surplus of up to 9 at.\% with respect to the target composition. The opposite trend is observed for $\alpha \geq 45^{\circ}$. Hence, the film composition while sputtering from a $\mathrm{Mo}_{2} \mathrm{BC}$ target is angle-dependent, which was previously observed by Olsen et al. [35] for sputtering (metallic) alloy targets. They explained mass-dependent angular distribution functions by backscattering of light elements on the heavier elements within the collision cascade in the target [35], resulting in an enrichment of lighter elements in directions normal to the target surface. Obviously, Mo cannot be backscattered due to reflective collisions with lighter elements, such as B and C.

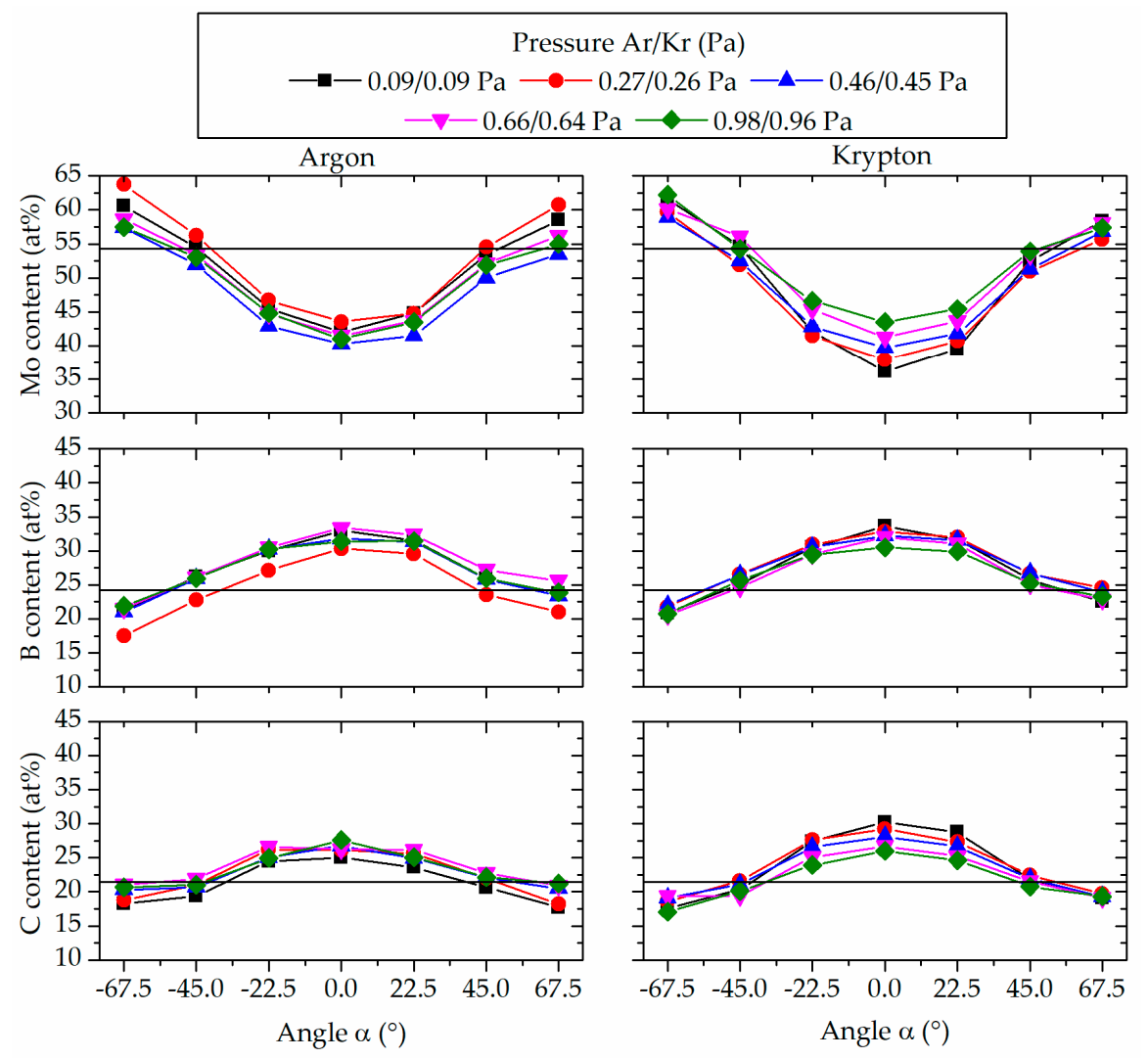

Figure 4. Angle-resolved composition evolution of the deposited thin films within the pressure range from 0.1 to $1.0 \mathrm{~Pa}$. The first pressure value pertains to the Ar depositions, the second value to the $\mathrm{Kr}$ depositions. The average oxygen content was less than 1.5 at.\% for all depositions and not considered further. The target composition is marked by the black horizontal lines. 
Comparing the Mo content of Ar and $\mathrm{Kr}$ depositions, a clear pressure-dependence can be seen for $\mathrm{Kr}$, while no significant composition changes were obtained for Ar. For Kr sputtering at $\alpha=0^{\circ}$, the Mo content changes from 36 at. $\%$ at $0.09 \mathrm{~Pa}$ to 43 at. $\%$ at $0.96 \mathrm{~Pa}$. The chemical variation at $\alpha= \pm 45^{\circ}$ is less distinct, while at $\alpha= \pm 67.5^{\circ}$, the Mo content variations are within the measurement error. For gas phase scattering of $\mathrm{B}$ and $\mathrm{C}$ in $\mathrm{Kr}$, the opposite trend is observed regarding the angle-dependent composition variation. However, the chemical variations due to pressure changes are within the measurement error. It is evident that an increase in pressure leads to a chemical composition closer to the nominal target composition and hence, stoichiometry. In an effort to determine the cause for the here observed sputtering gas-induced composition deviations, simulations were carried out, which allow for an independent analysis of composition deviations caused by sputtering of the target and scattering during the gas phase transport.

\subsection{Simulations}

The angle- and pressure-dependent film compositions with surface binding potentials (SBV's) determined by the energy conservation law and ab initio approaches, as discussed above, are presented in Figure 5 for depositions in Ar.

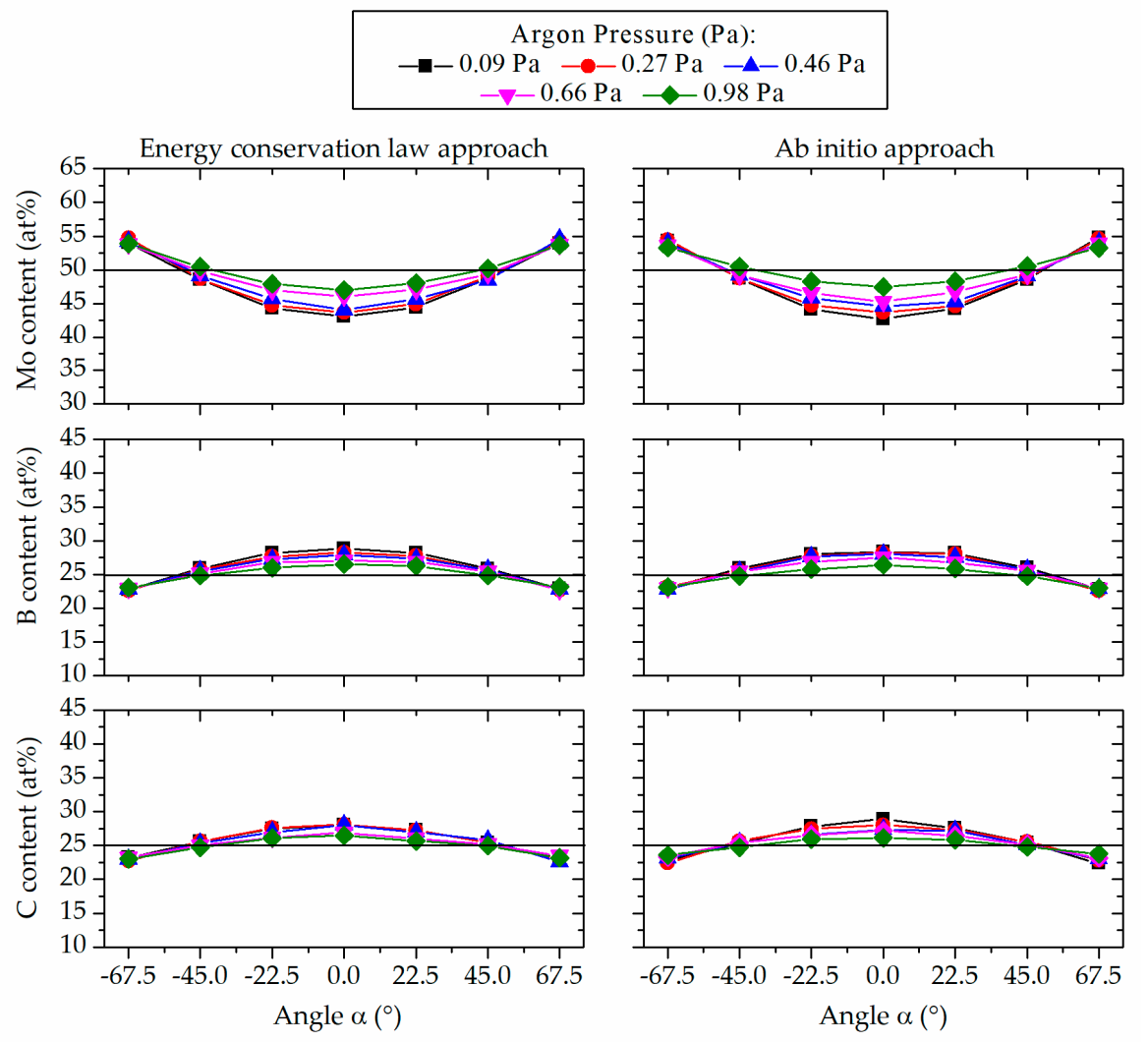

Figure 5. The simulated angle-resolved composition of thin films with the Ar pressure range from 0.09 to $0.98 \mathrm{~Pa}$. Considered surface binding energies of the two approaches (left) energy conservation law and (right) ab initio. The ideal stoichiometric target composition is marked by the black horizontal lines.

The trend of the experimentally determined angle- and pressure-dependent film composition depicted in Figure 4 is reproduced. The angle-dependence of Mo is convex, while B and C show a concave angle-dependence. Films at $\alpha \leq 22.5^{\circ}$ exhibit a deficiency of the heavy Mo and an enrichment of light $B$ and $C$. As in the experimental data for $\alpha>45^{\circ}$, an opposite trend is observed. The maximum difference in SBVs determined by the energy conservation law and ab initio approaches is $32 \%$. This SBV difference leads to composition differences of less or equal to 0.9 at. $\%$ and 1.1 at. $\%$ for Mo sputtered in $\mathrm{Ar}$ (Figure 5) and $\mathrm{Kr}$ (not shown), respectively. The magnitude of these composition 
differences cannot be resolved by EDX as the expected experimental errors are larger than the composition differences. For all simulations discussed below, SBVs determined by the ab initio approach were employed.

Pressure changes affect the target voltage and hence the ion energies impinging on the target (see Table 1). The influence of the ion energy on the ADF is illustrated in Figure 6. Within these simulations, scattering events during gas phase transport are deliberately not considered by utilizing an Ar pressure of $10^{-9} \mathrm{~Pa}$. Hence, these simulations only describe sputtering, specifically the effect of the kinetic energy of $\mathrm{Ar}^{+}$and $\mathrm{Kr}^{+}$on the angle-dependent composition of the sputtered flux. These simulations will therefore be referred to as initial ADFs. Increasing the kinetic energy of $\mathrm{Ar}^{+}$from 314 to $401 \mathrm{eV}$ (by $27 \%$ ) results in absolute mean composition differences of less than or equal to 0.4 at.\% for all simulations. Hence, the absolute, ion energy-induced composition changes in the sputtered flux are on average one order of magnitude smaller than the expected measurement error and hence could not be resolved by EDX measurements.

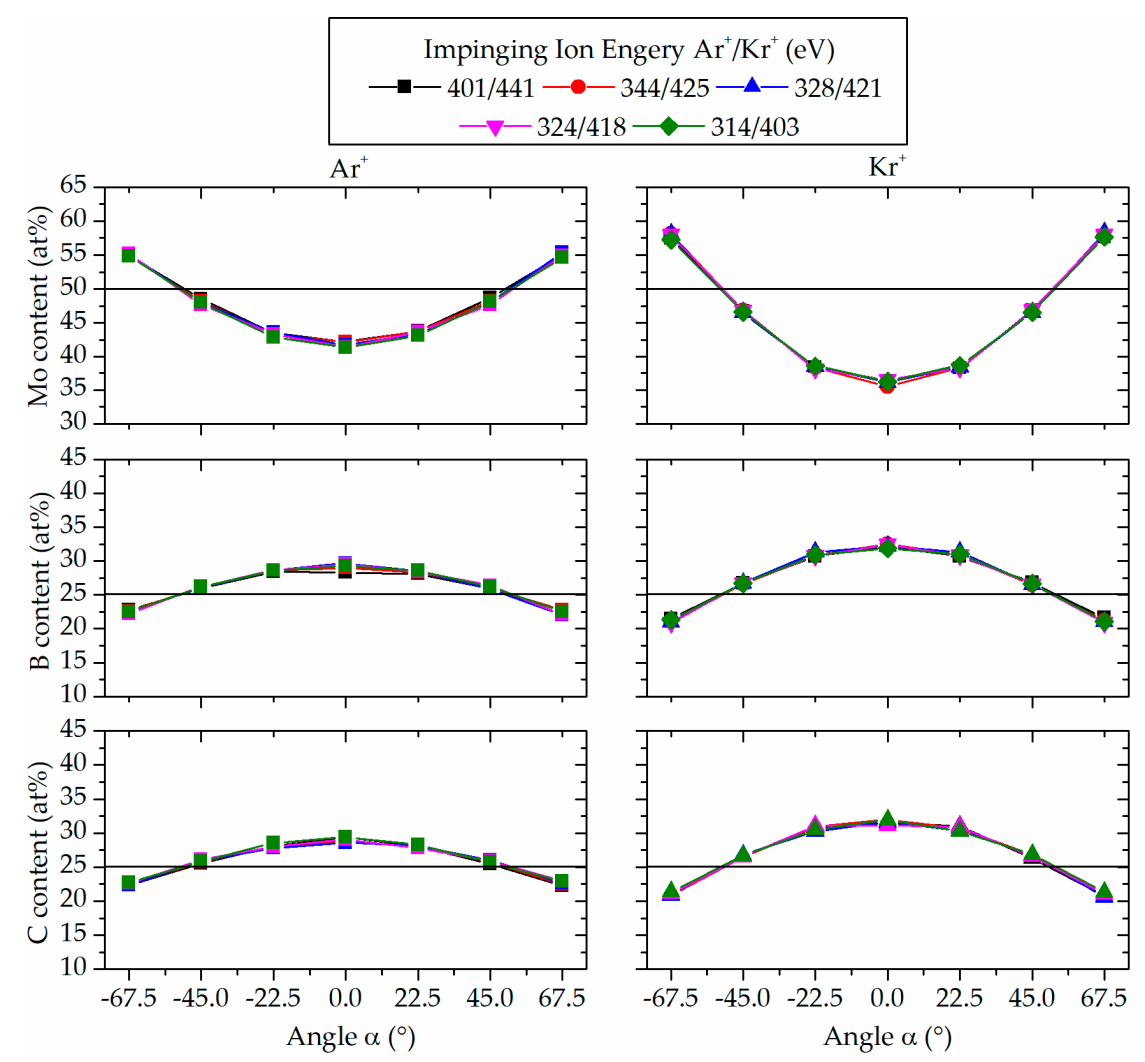

Figure 6. Angle-resolved composition evolution of the sputtered flux for different impinging ion energies of $\mathrm{Ar}^{+}$(left) and $\mathrm{Kr}^{+}$(right) ions. The first energy value pertains to $\mathrm{Ar}^{+}$sputtering, the second value to $\mathrm{Kr}^{+}$sputtering. The ideal stoichiometric target composition is marked by the black horizontal lines.

The initial ADF of Mo sputtered by $\mathrm{Ar}^{+}$(see Figure 6) exhibits a convex distribution, resulting in an Mo deficiency of 8 at. $\%$ at $\alpha=0^{\circ}$ with respect to a nominal Mo content of 50 at. $\%$. At $\alpha= \pm 67.5^{\circ}$, a surplus of 5 at.\% Mo is obtained. Both light elements exhibit a concave distribution, resulting in a surplus of 4 at. $\%$ at $\alpha=0^{\circ}$ and a deficiency of 3 at. $\%$ at $\alpha= \pm 67.5^{\circ}$ with respect to a nominal light element content of 25 at.\% each. Sputtering by $\mathrm{Kr}^{+}$(Figure 6) leads to more pronounced convex and concave distributions for heavy and light elements, respectively. The Mo deficiency and surplus are increased to 14 at. $\%$ and 8 at. $\%$, respectively. For both light elements, a surplus of 7 at. $\%$ and a deficiency of 4 at. $\%$ can be found at $\alpha=0^{\circ}$ and $\pm 67.5^{\circ}$, respectively. Compared to Ar, the sputtering-induced differences of $\mathrm{ADF}$ in $\mathrm{Kr}$ result in larger deviations between the composition of the target and the 
angle-dependent sputtered flux. These results can be rationalized based on the above discussed mass-dependent reflective collisions within the target surface. In the collision cascade, only B and C can be backscattered by Mo, leading to a preferential ejection of $\mathrm{B}$ and $\mathrm{C}$ close to the target normal. Mo cannot be backscattered due to a reflective collision with lighter B or C.

Simulations of the film composition that take, in addition to sputtering at the target, the scattering events within gas phase transport into account, are shown in Figure 7. The Ar or Kr pressures are identical to the experimental pressures depicted in Table 1. Generally, the experimentally-determined angle-dependent film composition data are consistent with the simulation results.

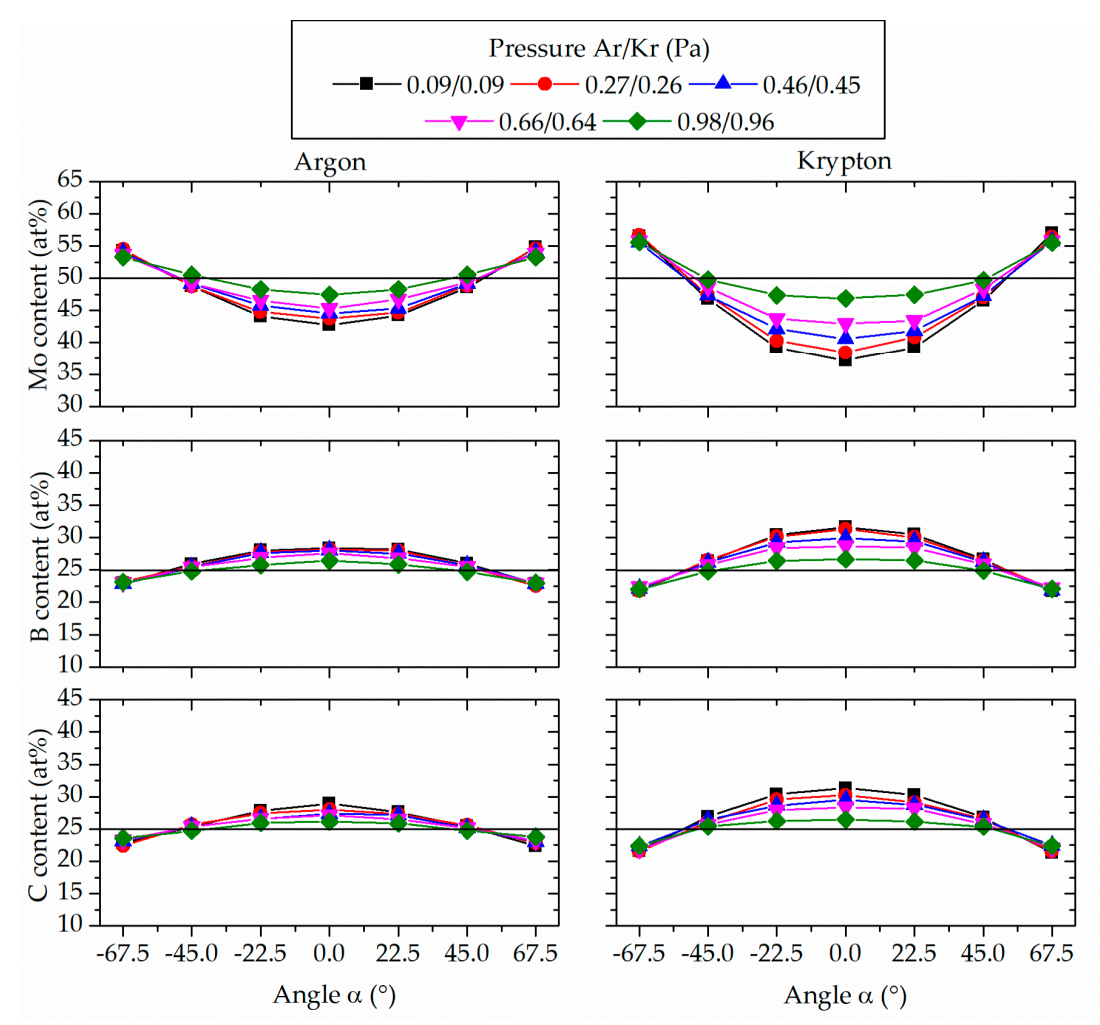

Figure 7. Angle-resolved evolution of simulated film compositions considering sputtering at the target, as well as scattering during gas phase transport. The first pressure value pertains to the Ar depositions, the second value to the $\mathrm{Kr}$ depositions. The ideal stoichiometric target composition is marked by the black horizontal lines.

Significant differences between the initial ADF and the ADF obtained after scattering during transport in the gas phase are obtained for $\mathrm{Ar}$ and $\mathrm{Kr}$ as the pressure is increased by one order of magnitude. An increase in Mo content at $\alpha=0^{\circ}$ of 4.7 at. $\%$ and 9.7 at. $\%$ and for both light elements a decrease of 3 at. $\%$ and 5 at. $\%$ can be obtained in Ar and $\mathrm{Kr}$, respectively. At $\alpha= \pm 67.5^{\circ}$, no significant pressure-induced impact on the chemical composition can be observed. Generally, the pressure-induced variations in chemical composition are more pronounced in $\mathrm{Kr}$ and are in good agreement with the experimentally-determined data. Comparison to the EDX composition measurement error indicates that the pressure-dependent composition variations simulated in $\mathrm{Ar}$ cannot be resolved experimentally.

To identify the cause of the here discussed angle- and pressure-dependent film composition variations, the angle-resolved average trajectory lengths of the sputtered species are calculated. The average trajectory length, $d$, is the mean distance a particle travels from sputtering at the target to deposition at the substrate surface and is maximized for scattering events at large scattering angles and short mean free paths. The pressure-dependence of $d$ is shown in Figure 8 for Ar and Kr. 


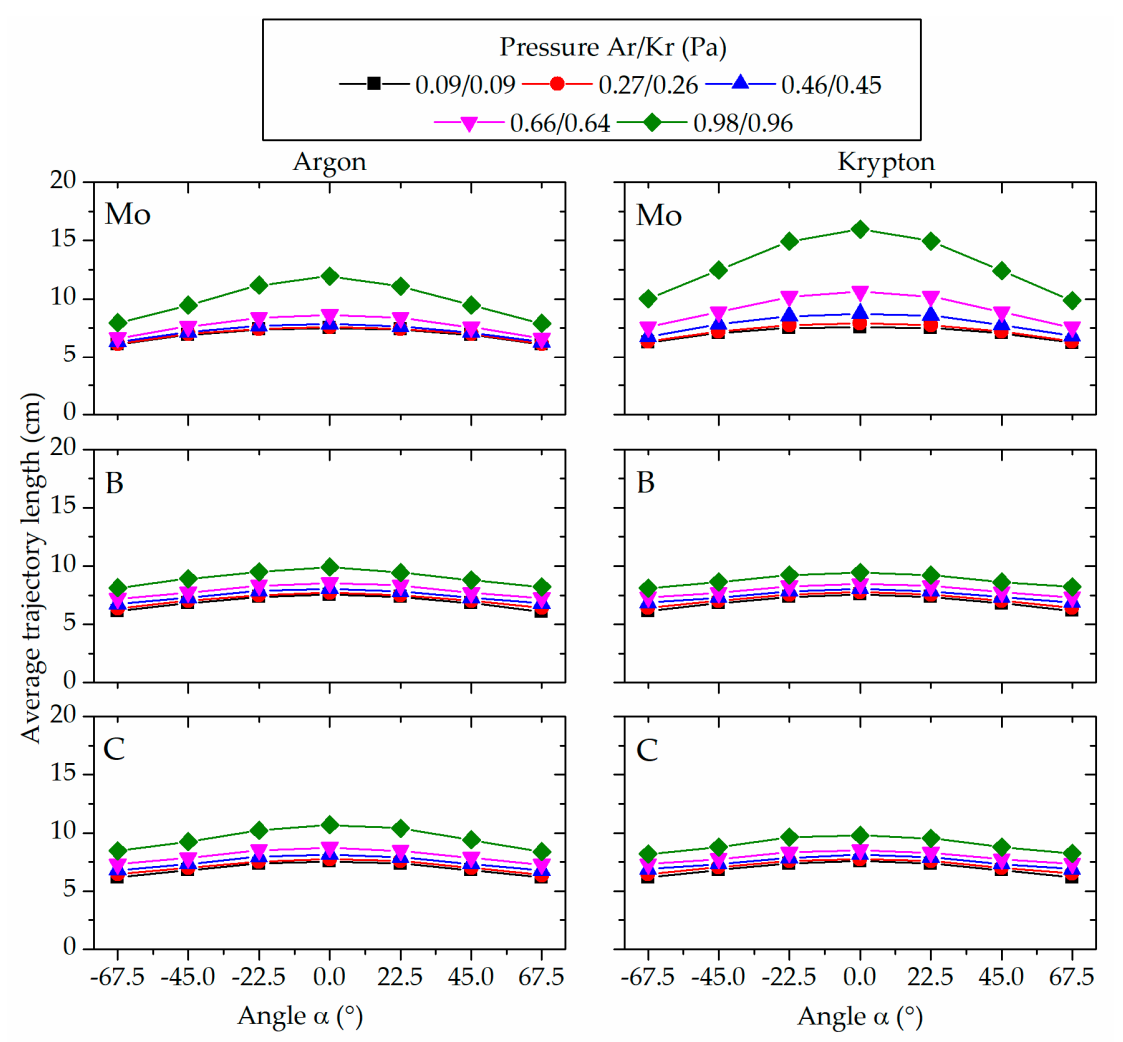

Figure 8. Simulated average trajectory length of the sputtered atoms transported through the gas phase at given $\mathrm{Ar}$ (left) and Krypton (right) pressures. The first pressure value pertains to the Ar depositions, the second value to the $\mathrm{Kr}$ depositions.

Increasing the Ar pressure by one order of magnitude results in a relative increase of $d$ at $\alpha=0^{\circ}$ of $59 \%, 31 \%$, and $42 \%$ for Mo, B, and C, respectively. The same change in $\mathrm{Kr}$ pressure results in a relative increase of $\mathrm{d}$ at $\alpha=0^{\circ}$ of $111 \%, 25 \%$, and $29 \%$ for Mo, B, and C, respectively. Hence, the average Mo trajectory length is up to $34 \%$ larger in $\mathrm{Kr}$ than in Ar.

The average number of Mo collisions at the maximum Ar and $\mathrm{Kr}$ pressures at $\alpha=0^{\circ}$ is 19.4 and 22.9, respectively, exhibiting a relative difference of $18.2 \%$. As the pressure-induced increase in average trajectory length $d$ is caused by the number of collisions, as well as the average scattering angle, simulations were conducted where the number of collisions was kept constant to unravel the contribution of the average scattering angle. For each element, one additional simulation was conducted at a specific $\mathrm{Kr}$ pressure (0.89 Pa for Mo and 1.10 Pa for B and C) to match the number of collisions computed for scattering in $0.98 \mathrm{~Pa}$ of Ar, which are 19.4, 10.7, and 12.4, for Mo, B, and $C$, respectively.

At a constant average number of Mo collisions of 19.4, the pressure-induced increase in $d$ of Mo is 23\% larger in $\mathrm{Kr}$ than in Ar. At an average number of collisions of 10.7 and 12.4 for B and C, respectively, a pressure-induced increase in $\mathrm{d}$ of $1.3 \%$ and $0.7 \%$ was obtained for $\mathrm{B}$ and $\mathrm{C}$, respectively. Hence, it is deduced that the average scattering angle of Mo is significantly larger in $\mathrm{Kr}$ than in $\mathrm{Ar}$ and that the evolution of the angle- and pressure-dependent film composition is determined by the average scattering angle of Mo. Assuming energy and momentum conservation, a mass-dependent expression for the maximum scattering angle of a particle with a mass larger than the gas species is given by Equation (6) $[47,48]$, where $\vartheta_{\max }$ is the maximum scattering angle and $m_{\mathrm{Mo}}$ and $m_{i}$ are the masses of Mo and the gas atom, respectively. Consequently, maximum scattering angles for Mo of $24.6^{\circ}$ and $60.4^{\circ}$ in $\mathrm{Ar}$ and $\mathrm{Kr}$, respectively, were obtained for the masses of 95.96, 39.95, and $83.80 \mathrm{amu}$ 
for Mo, Ar, and Kr, respectively [49]. Hence, the above deduced larger average scattering angle for Mo in $\mathrm{Kr}$ as compared to Ar is caused by the mass ratio between the sputtering gas and Mo.

$$
\vartheta_{\max }=\arcsin \left(\frac{m_{\mathrm{gas}}}{m_{\mathrm{Mo}}}\right)
$$

The simulations carried out within this work allowed an independent consideration of the sputtering process at the target surface, as well as the scattering events within the gas phase transport. Pressure variations over one order of magnitude insignificantly influence the sputtering process, whereas the mass of the impinging ion exhibits a strong impact on the initial ADF. Sputtering-induced differences between the target and thin film composition caused by $\mathrm{Kr}^{+}$are larger compared to sputtering with $\mathrm{Ar}^{+}$, which is in agreement with the sputtering experiments at low pressures.

Gas phase scattering events induced-variations in film chemical composition depend on both the gas pressure and mass of the gas atom. The average trajectory length was shown to be a good indicator for the impact of scattering. To unravel the relative contribution of numbers of collision and average scatter angle, simulations with an identical number of collisions in $\mathrm{Ar}$ and $\mathrm{Kr}$ of 19.4, 10.7, and 12.4, for Mo, B, and C, respectively, were conducted. In $\mathrm{Kr}$ compared to Ar a dominant pressure-induced increase in $d$ of $23 \%$ for Mo, compared to $1.3 \%$ and $0.7 \%$ for B, and C, respectively, was obtained. Hence, the significantly larger average trajectory length of $\mathrm{Mo}$ in $\mathrm{Kr}$ as compared to $\mathrm{Ar}$ at the same number of collisions can be rationalized by the larger average scattering angle of Mo, which in turn controls the evolution of the angle- and pressure-dependent film composition.

\section{Conclusions}

The evolution of the angle-resolved composition of Mo-B-C thin films deposited from a $\mathrm{Mo}_{2} \mathrm{BC}$ compound target was investigated experimentally and theoretically as a function of the Ar and $\mathrm{Kr}$ pressure. Samples were positioned in a specific angular arrangement from $\alpha=0^{\circ}$ to $\pm 67.5^{\circ}$ with respect to the target normal with a fixed target substrate distance.

Considering the simulated mass-dependent initial angular distribution functions, a convex distribution for Mo was observed, whereas $B$ and $C$ exhibited concave distributions as a consequence of reflective collisions in the collision cascade. B and C can only be backscattered by the heavy Mo leading to the preferential ejection of $B$ and $C$ close to the target normal. Obviously, Mo cannot be backscattered due to a reflective collision with a lighter element.

Within experiments and simulations, the observed change in angle-resolved composition as a result of a by one order of magnitude increased Ar pressure was lower than the expected measurement error and hence, cannot be resolved by EDX. On the contrary, sputtering by $\mathrm{Kr}^{+}$results in significantly larger deviations between the target and the film composition. These deviations can be rationalized based on reflective collisions in the collision cascade. As the $\mathrm{Kr}$ pressure is increased, scattering during transport in the gas phase results in angular resolved compositions that approach the target composition. Furthermore, based on considering the relative contributions of the number of collisions and scatter angle to the average trajectory length, it is inferred that the significantly larger average trajectory length of $\mathrm{Mo}$ in $\mathrm{Kr}$ compared to $\mathrm{Ar}$ can be rationalized by an on average larger scattering angle of Mo. It is shown that the mass ratio between sputtering gas and sputtered species defines the scattering angle within the collision cascades in the target, as well as for the collisions in the gas phase, which in turn define the angle- and pressure-dependent film compositions.

Author Contributions: Conceptualization, J.-O.A., S.M. and J.M.S.; Methodology, J.-O.A., S.M. and D.P.; Investigation, J.-O.A., S.M. and D.P.; Writing, J.-O.A., S.M., D.P. and J.M.S.; Project administration, J.-O.A., S.M., J.M.S. and J.-O.A.; Funding acquisition, J.M.S.

Funding: The authors gratefully acknowledge the financial support granted by the DFG SCHN 735/35-1. 
Acknowledgments: The authors gratefully acknowledge G. Dehm for fruitful discussions. The simulations were performed with computing resources granted by JARAHPC from RWTH Aachen University under Project No. JARA0131. Support by VR-RFI (contracts \#821-2012-5144 \& \#2017-00646_9) and the Swedish Foundation for Strategic Research (SSF, contract RIF14-0053) supporting accelerator operation at Uppsala University is gratefully acknowledged.

Conflicts of Interest: The authors declare no conflict of interest.

\section{References}

1. Bovin, J.O.; O'Keeffe, M.; Stenberg, L. Planar defects in $\mathrm{Mo}_{2}$ BC. An electron microscope study. J. Solid State Chem. 1977, 22, 221-231. [CrossRef]

2. Jeitschko, W.; Nowotny, H.; Benesovsky, F. Die kristallstruktur von $\mathrm{Mo}_{2} \mathrm{BC}$. Monatshefte für Chemie und verwandte Teile anderer Wissenschaften 1963, 94, 565-568. [CrossRef]

3. Emmerlich, J.; Music, D.; Braun, M.; Fayek, P.; Munnik, F.; Schneider, J.M. A proposal for an unusually stiff and moderately ductile hard coating material: $\mathrm{Mo}_{2}$ BC. J. Phys. D Appl. Phys. 2009, 42, 185406. [CrossRef]

4. Bolvardi, H.; Emmerlich, J.; Music, D.; von Appen, J.; Dronskowski, R.; Schneider, J.M. Systematic study on the electronic structure and mechanical properties of $X_{2} \mathrm{BC}(X=\mathrm{Mo}, \mathrm{Ti}, \mathrm{V}, \mathrm{Zr}, \mathrm{Nb}, \mathrm{Hf}$, Ta and W). J. Phys. Condens. Matter 2012, 25, 045501. [CrossRef]

5. Bolvardi, H.; Emmerlich, J.; Mráz, S.; Arndt, M.; Rudigier, H.; Schneider, J.M. Low temperature synthesis of $\mathrm{Mo}_{2} \mathrm{BC}$ thin films. Thin Solid Films 2013, 542, 5-7. [CrossRef]

6. Sarakinos, K.; Alami, J.; Konstantinidis, S. High power pulsed magnetron sputtering: A review on scientific and engineering state of the art. Surf. Coat. Technol. 2010, 204, 1661-1684. [CrossRef]

7. Ohring, M. Materials Science of Thin Films, 2nd ed.; Academic Press Limited: Cambridge, MA, USA, 1992.

8. Emmerlich, J.; Högberg, H.; Sasvári, S.; Persson, P.O.; Hultman, L.; Palmquist, J.P.; Jansson, U.; Molina-Aldareguia, J.M.; Czigány, Z. Growth of $\mathrm{Ti}_{3} \mathrm{SiC}_{2}$ thin films by elemental target magnetron sputtering. J. Appl. Phys. 2004, 96, 4817-4826. [CrossRef]

9. Eklund, P.; Joelsson, T.; Ljungcrantz, H.; Wilhelmsson, O.; Czigány, Z.; Högberg, H.; Hultman, L. Microstructure and electrical properties of Ti-Si-C-Ag nanocomposite thin films. Surf. Coat. Technol. 2007, 201, 6465-6469. [CrossRef]

10. Chen, L.; Holec, D.; Du, Y.; Mayrhofer, P.H. Influence of Zr on structure, mechanical and thermal properties of Ti-Al-N. Thin Solid Films 2011, 519, 5503-5510. [CrossRef] [PubMed]

11. Eklund, P.; Beckers, M.; Jansson, U.; Högberg, H.; Hultman, L. The $\mathrm{M}_{n+1} \mathrm{AX} X_{n}$ phases: Materials science and thin-film processing. Thin Solid Films 2010, 518, 1851-1878. [CrossRef]

12. Neidhardt, J.; Mráz, S.; Schneider, J.M.; Strub, E.; Bohne, W.; Liedke, B.; Moller, W.; Mitter, C. Experiment and simulation of the compositional evolution of Ti-B thin films deposited by sputtering of a compound target. J. Appl. Phys. 2008, 104, 063304. [CrossRef]

13. Mitterer, C. Borides in thin film technology. J. Solid State Chem. 1997, 133, 279-291. [CrossRef]

14. Kunc, F.; Musil, J.; Mayrhofer, P.H.; Mitterer, C. Low-stress superhard Ti-B films prepared by magnetron sputtering. Surf. Coat. Technol. 2003, 174, 744-753. [CrossRef]

15. Shaginyan, L.R.; Mišina, M.; Kadlec, S.; Jastrabik, L.; Mackova, A.; Peřina, V. Mechanism of the film composition formation during magnetron sputtering of WTi. J. Vac. Sci. Technol. A 2001, 19, 2554-2566. [CrossRef]

16. Jonsson, L.B.; Hedlund, C.; Katardjiev, I.V.; Berg, S. Compositional variations of sputter deposited Ti/W barrier layers on substrates with pronounced surface topography. Thin Solid Films 1999, 348, $227-232$. [CrossRef]

17. Ramarotafika, H.; Lemperiere, G. Influence of a d.c. substrate bias on the resistivity, composition, crystallite size and microstrain of WTi and WTi-N films. Thin Solid Films 1995, 266, 267-273. [CrossRef]

18. Rogers, B.R.; Tracy, C.J.; Cale, T.S. Compositional variation in sputtered Ti-W films due to re-emission. J. Vac. Sci. Technol. A 1994, 12, 2980-2984. [CrossRef]

19. Rogers, B.R.; Cale, T.S.; Chang, Y.K. Simulation and experimental study of re-emission during sputter deposition of Ti-W films. J. Vac. Sci. Technol. A 1996, 14, 1142-1146. [CrossRef]

20. Bergstrom, D.B.; Tian, F.; Petrov, I.; Moser, J.; Greene, J.E. Origin of compositional variations in sputter-deposited $\mathrm{Ti}_{x} \mathrm{~W}_{1-x}$ diffusion barrier layers. Appl. Phys. Lett. 1995, 67, 3102-3104. [CrossRef] 
21. Rossnagel, S.M.; Yang, I.; Cuomo, J.J. Compositional changes during magnetron sputtering of alloys. Thin Solid Films 1991, 199, 59-69. [CrossRef]

22. Dirks, A.G.; Wolters, R.A.M.; Nellissen, A.J.M. On the microstructure-property relationship of W-Ti-(N) diffusion barriers. Thin Solid Films 1990, 193, 201-210. [CrossRef]

23. Willer, J.; Pompl, S.; Ristow, D. Sputter-deposited WB $\mathrm{W}_{x}$ films. Thin Solid Films 1990, 188, 157-163. [CrossRef]

24. Simao, R.A.; Costa, A.K.; Achete, C.A.; Camargo Jr, S.S. Magnetron sputtering SiC films investigated by AFM. Thin Solid Films 2000, 377, 490-494. [CrossRef]

25. Murakami, Y.; Shingyoji, T. Compositional difference between films and targets in sputtering of refractory metal silicides. J. Vac. Sci. Technol. A 1990, 8, 851-854. [CrossRef]

26. Yamazaki, T.; Ikeda, N.; Tawara, H.; Sato, M. Investigation of composition uniformity of MoSi $x_{x}$ sputtering films based on measurement of angular-distribution of sputtered atoms. Thin Solid Films 1993, 235, 71-75. [CrossRef]

27. Liao, M.Y.; Gotoh, Y.; Tsuji, H.; Ishikawa, J. Deposition of vanadium carbide thin films using compound target sputtering and their field emission. J. Vac. Sci. Technol. A 2005, 23, 1379-1383. [CrossRef]

28. Liao, M.Y.; Gotoh, Y.; Tsuji, H.; Ishikawa, J. Compound-target sputtering for niobium carbide thin-film deposition. J. Vac. Sci. Technol. B 2004, 22, L24-L27. [CrossRef]

29. Mráz, S.; Emmerlich, J.; Weyand, F.; Schneider, J.M. Angle-resolved evolution of the composition of Cr-Al-C thin films deposited by sputtering of a compound target. J. Phys. D Appl. Phys. 2013, 46, 135501. [CrossRef]

30. Rueß, H.; to Baben, M.; Mráz, S.; Shang, L.; Polcik, P.; Kolozsvári, S.; Hans, M.; Primetzhofer, D.; Schneider, J.M. HPPMS deposition from composite targets: Effect of two orders of magnitude target power density changes on the composition of sputtered Cr-Al-C thin films. Vacuum 2017, 145, 285-289. [CrossRef]

31. Walter, C.; Martinez, C.; El-Raghy, T.; Schneider, J.M. Towards large area MAX phase coatings on steel. Steel Res. Int. 2005, 76, 225-228. [CrossRef]

32. Palmquist, J.P.; Jansson, U.; Seppänen, T.; Persson, P.; Birch, J.; Hultman, L.; Isberg, P. Magnetron sputtered epitaxial single-phase $\mathrm{Ti}_{3} \mathrm{SiC}_{2}$ thin films. Appl. Phys. Lett. 2002, 81, 835-837. [CrossRef]

33. Eklund, P.; Beckers, M.; Frodelius, J.; Högberg, H.; Hultman, L. Magnetron sputtering of $\mathrm{Ti}_{3} \mathrm{SiC}_{2}$ thin films from a compound target. J. Vac. Sci. Technol. A 2007, 25, 1381-1388. [CrossRef]

34. Jo, Y.H.; Mohanty, B.C.; Yeon, D.H.; Lee, S.M.; Cho, Y.S. Single elementary target-sputtered $\mathrm{Cu}_{2} \mathrm{ZnSnSe}_{4}$ thin film solar cells. Sol. Energy Mater. Sol. Cells 2015, 132, 136-141. [CrossRef]

35. Olson, R.R.; King, M.E.; Wehner, G.K. Mass effects on angular distribution of sputtered atoms. J. Appl. Phys. 1979, 50, 3677-3683. [CrossRef]

36. Van Aeken, K.; Mahieu, S.; Depla, D. The metal flux from a rotating cylindrical magnetron: A Monte Carlo simulation. J. Phys. D Appl. Phys. 2008, 41, 205307. [CrossRef]

37. Möller, W.; Eckstein, W.; Biersack, J.P. Tridyn-binary collision simulation of atomic collisions and dynamic composition changes in solids. Comput. Phys. Commun. 1988, 51, 355-368. [CrossRef]

38. Möller, W.; Posselt, M. TRIDYN_FZR user manual. Qucosa 2001.

39. Chase, M.W., Jr.; Davies, C.A.; Downey, J.R.; Frurip, D.J.; McDonald, R.A.; Syverud, A.N. JANAF thermochemical tables, 3rd ed. J. Phys. Chem. Ref. Data 1985, 14 (Suppl. 1).

40. Kresse, G.; Hafner, J. Ab initio molecular dynamics for open-shell transition metals. Phys. Rev. B 1993, 48, 13115. [CrossRef]

41. Kresse, G.; Hafner, J. Ab initio molecular-dynamics simulation of the liquid-metal-amorphoussemiconductor transition in germanium. Phys. Rev. B 1994, 49, 14251. [CrossRef]

42. Perdew, J.P.; Burke, K.; Ernzerhof, M. Generalized gradient approximation made simple. Phys. Rev. Lett. 1996, 77, 3865. [CrossRef]

43. Kresse, G.; Joubert, D. From ultrasoft pseudopotentials to the projector augmented-wave method. Phys. Rev. B 1999, 59, 1758. [CrossRef]

44. Blöchl, P.E. Projector augmented-wave method. Phys. Rev. B 1994, 50, 17953. [CrossRef]

45. Monkhorst, H.J.; Pack, J.D. Special points for Brillouin-zone integrations. Phys. Rev. B 1976, $13,5188$. [CrossRef]

46. Momma, K.; Izumi, F. VESTA 3 for three-dimensional visualization of crystal, volumetric and morphology data. J. Appl. Crystallogr. 2011, 44, 1272-1276. [CrossRef] 
47. McDaniel, E.W. Collision Phenomena in Ionized Gases; Wiley: New York, NY, USA, 1964.

48. Behrisch, R. Sputtering by Particle Bombardment I; Springer: Berlin, Germany, 1981.

49. Wieser, M.E.; Berglund, M. Atomic weights of the elements 2007 (IUPAC technical report). Pure Appl. Chem. 2009, 81, 2131-2156. [CrossRef] 